\title{
Collisional line shapes for low frequency vibrations of adsorbates on a metal surface
}

\author{
J. L. Vega, R. Guantes, and S. Miret-Artés \\ Instituto de Matemáticas y Física Fundamental, Consejo Superior de Investigaciones Científicas, \\ Serrano 123, 28006 Madrid, Spain \\ D. A. Micha \\ Quantum Theory Project, Departments of Chemistry and Physics, University of Florida, Gainesville, \\ Florida 32611-8435
}

(Received 21 January 2004; accepted 9 August 2004)

\begin{abstract}
The dynamics of atoms or molecules adsorbed on a metal surface, and excited by collisions with an atomic beam, are treated within a theory that includes energy dissipation into lattice vibrations by means of a frequency and temperature dependent friction function. The theory provides dynamic structure factors for energy transfer derived from collisional time correlation functions. It describes the relaxation of a vibrationally excited atom or molecule within a model of a damped quantum harmonic oscillator bilinearly coupled to a bath of lattice oscillators. The collisional time correlation function is generalized to include friction effects and is applied to the vibrational relaxation of the frustrated translation mode of $\mathrm{Na}$ adsorbed on a $\mathrm{Cu}(001)$ surface, $\mathrm{CO}$ on $\mathrm{Cu}(001)$, and $\mathrm{CO}$ on $\mathrm{Pt}(111)$, following excitation by collisions with He atoms. Results for the frequency shift and width of line shapes versus surface temperature are in very good agreement with experimental measurements of inelastic $\mathrm{He}$ atom scattering. Our interpretation of the experimental results provides insight on the relative role of phonon versus electron-hole relaxation. (C) 2004 American Institute of Physics. [DOI: 10.1063/1.1802274]
\end{abstract}

\section{INTRODUCTION}

Vibrational spectroscopy of adsorbed species (atoms or molecules) on solid surfaces is a powerful technique for extracting information on adsorbate-adsorbate and adsorbatesubstrate interactions governing elementary processes such as capture, desorption and dissociation, and complex processes such as diffusion and surface reactions. The vibrational modes of adspecies can be classified according to the magnitude of their frequencies as internal modes (with high frequencies) and external or librational modes (with low frequencies). ${ }^{1}$ The first ones are localized inside the adsorbed species, and their damping on metals is mostly via electronhole pair excitations, which are practically temperature independent. ${ }^{2}$ The external modes correspond to some frustrated motion as a whole with respect to the surface (for example, for a molecule this would be a frustrated rotation or vibration or translational motion) and can exchange energy with surface phonon modes in a temperature dependent damping mechanism or with electron-hole pairs. ${ }^{3,4}$ The corresponding vibrational energies have been determined by different techniques such as inelastic neutron ${ }^{5,6}$ and electron ${ }^{7}$ scattering, infrared spectroscopy ${ }^{8}$ and, more recently, inelastic helium atom scattering (IHAS). ${ }^{1}$ In particular, the first measurements of a frustrated translational $(T)$ mode was carried out by Lahee et al. using IHAS several years ago ${ }^{9}$ for the system $\mathrm{CO} / \mathrm{Cu}(001)$, and were interpreted by them and others in terms of phonon excitations. Clearly, characterization of the vibrational damping of the $T$-mode is an important issue because it controls surface processes such as adsorbate diffusion, sliding and/or lubricant behaviors at microscopic atomic scale. These processes are related to vibrational lifetimes and can be attributed to both phononic and electronic frictional damping, ${ }^{4,10}$ although the relative importance of these two mechanisms is not usually known. The rate of energy exchange or friction coefficient is given by the inverse of the corresponding adsorbate vibrational lifetime as obtained from line shapes.

In general, natural line shapes have small line widths and cannot be observed without special techniques because they are concealed by other broadening effects. In damping processes, the existence of alternative relaxation channels shorten the lifetime of a particular process and therefore result in line broadening. Roughly speaking, we have two types of broadening of adsorbate spectral lines, homogeneous and inhomogeneous. The first one occurs when all the species are in the same initial state and leads typically to Lorentzian line shapes. On the contrary, the inhomogeneous broadening takes place when each species is in a different initial state and leads usually to Gaussian line shapes with a width many times greater than a Lorentzian one. This broadening consists of a superposition of many individual, homogeneous broadened lines which merge into a single broadened line. In the vibrational damping process occurring at surfaces, of present interest, the adsorbate coverage leads to inhomogeneous broadening effects and vibrational frequency dispersion. However, if the coverage is very low, the vibrational modes are dispersionless indicating the absence of adsorbate-adsorbate interactions and homogeneous broadening is then expected. Previous theoretical works on vibrational relaxation of nonlinear oscillators in liquids ${ }^{11}$ or 
coupled to a linear bath ${ }^{12}$ can be found in the literature within the generalized Langevin equation formalism. For a recent review on general vibrational relaxation see, for example, Refs. 13 and 14.

For the frustrated translation or $T$-mode of atoms or molecules on metal surfaces, it is known that vibrational damping is not an activated process like diffusion and the $T$-mode can be considered dispersionless for very low coverage. In principle, a driven damped harmonic oscillator is a good model for the description of the $T$-motion as probed by $\mathrm{He}$ atom beams. Using IHAS for the $\mathrm{Na} / \mathrm{Cu}(001)$ system, it has been observed ${ }^{10}$ that as the surface temperature increases, the $T$-peak shifts towards lower frequencies and broadens almost linearly in a region between $0 \mathrm{~K}$ and $200 \mathrm{~K}$. Furthermore, as the collisional momentum transfer increases, the intensity of the inelastic $T$-peak increases at the expense of the quasielastic peak because of coupling of diffusion and vibrational motions. The experimental line shapes were assumed to be Lorentzian functions of the energy transfer. At low surface temperatures, the full width at half maximum (FWHM) of the $T$-peak has been interpreted to be dominated by frictional damping (characterized by the nonadiabatic coupling to the substrate excitations, phonons, and electron-hole pairs) whereas, at high temperatures, anharmonicity has been expected to be the dominant effect. Finally, the $T$-mode lifetime has been obtained by (linear) extrapolation to zero temperature to obtain the inverse of the friction coefficient. In addition, results of a Langevin dynamics simulation ${ }^{10}$ of the $T$-motion furnish a very good agreement with the experimental results. This is surprising because the $T$-motion is of quantum nature, while the numerical simulations do not include the quantum behavior. Similar arguments can be given for $\mathrm{CO} / \mathrm{Cu}(001)$ and $\mathrm{CO} / \mathrm{Pt}(111)$ systems. ${ }^{10}$

Progress has been made on a classical theory of IHAS together with quantum corrections ${ }^{15}$ but a complete quantal treatment is still lacking and should be developed to provide a better or alternative interpretation of the experimental results. Line shape shifts and widths and, in general, broadening effects are important questions which need an adequate theoretical interpretation. A simple model containing the main physical features can be constructed from considerations of time scales and energetics during collisional excitation and the following relaxation of vibrationally excited adsorbate states. As we will describe later on, for He scattering from the above mentioned three systems, collision times at the experimental projectile energies are much shorter than the average diffusion time, so that the model can ignore diffusion and concentrate on the effect of energy dissipation on the line shape. Furthermore, estimated collision times at the low projectile energy are appreciably longer than typical electronic density fluctuation times (or electron-hole lifetimes), indicating that during most of the collision the adsorbate is interacting with the lattice vibrations, after the electron fluctuations have settled down. ${ }^{16}$ Therefore the following model concentrates on the effect of lattice vibrations. Moreover, in this work, our purpose is to provide a quantal model suitable for atoms/molecules adsorbed on metal surfaces, relating energy transfer cross sections to a dynamical structure factor obtained from the Fourier trans- form of a collisional time-correlation function (TCF). ${ }^{6,17,18}$ We derive compact expressions for the TCFs from a model Hamiltonian which incorporates the coupling of a vibrating adsorbed particle with the lattice vibrations of the substrate metal to describe energy dissipation and how it leads to a line shift and linewidth related to a friction coefficient. This is shown in Sec. II, which makes use of the algebra of vibrational creation and annihilation operators. ${ }^{19}$ Section III applies the treatment to the excitation and relaxation of the $T$-mode of the $\mathrm{Na} / \mathrm{Cu}(001), \mathrm{CO} / \mathrm{Cu}(001)$, and $\mathrm{CO} / \mathrm{Pt}(111)$ systems by means of a fitting procedure to experimental values at a single temperature to obtain the line shift and widths of the $T$-peak versus the whole range of measured surface temperatures. Finally, in Sec. IV, some comments on alternative mechanisms presently proposed to explain the origin and magnitudes of line shapes are presented.

\section{QUANTUM TREATMENT OF VIBRATIONAL RELAXATION}

\section{A. Dynamic structure factor}

The quantity measured in quasielastic He atom scattering experiments is the differential reflection probability for a projectile helium atom scattered into a solid angle $\Omega$ with an energy exchange $\hbar \omega$, which is given by the following expression: ${ }^{20,21}$

$$
\begin{aligned}
\frac{d^{2} R(\mathbf{K}, \omega)}{d \Omega d \omega} & =n_{A} F_{\mathrm{He} A}^{2} \iint G(\mathbf{R}, t) e^{i(\mathbf{K} \cdot \mathbf{R}-\omega t)} d \mathbf{R} d t \\
& =n_{A} F_{\mathrm{HeA}}^{2} S(\mathbf{K}, \omega),
\end{aligned}
$$

where $\mathbf{K}$ is the wave vector transfer parallel to the surface involving the initial and final scattering angles and momenta and related by the kinematic condition

$$
\mathbf{K}=\mathbf{k}_{f} \sin \theta_{f}-\mathbf{k}_{i} \sin \theta_{i},
$$

and the scattering energy transfer is given by

$$
\hbar \omega=\hbar^{2}\left(k_{f}^{2}-k_{i}^{2}\right) /\left(2 m_{\mathrm{He}}\right),
$$

$n_{A}$ is the adsorbate concentration on the surface and $F_{\mathrm{HeA}}$ is the atomic form factor depending on the interaction potential between the He projectile and a single adsorbate $A$. Moreover, $G(\mathbf{R}, t)$ is the so-called time-dependent pair correlation function and it gives the ensemble averaged probability density for finding an adparticle at the surface position $\mathbf{R}$ at time $t$, given that an adparticle is at the origin at some arbitrary time $t=0$. At low concentrations, when interactions among adsorbates can be ignored, the G-function can be described by its low density limit, ${ }^{22}$ the so-called self-component. The Fourier transform from space and time of the $G$-function to momentum and frequency is known as the dynamic structure factor $S(\mathbf{K}, \omega)$. In what follows we will also use the partial Fourier transform from space to wave vector variables, called the intermediate scattering function $I(\mathbf{K}, t)$, which is related to the dynamic structure factor by 


$$
\begin{aligned}
S(\mathbf{K}, \omega) & =\frac{1}{2 \pi} \int_{-\infty}^{\infty} e^{-i \omega t} I(\mathbf{K}, t) d t \\
& =\frac{1}{2 \pi} \int_{-\infty}^{\infty} d t e^{-i \omega t}\left\langle e^{-i \mathbf{K} \cdot \mathbf{R}(0)} e^{i \mathbf{K} \cdot \mathbf{R}(t)}\right\rangle,
\end{aligned}
$$

where the brackets in the second integral denote an ensemble average. The dynamic structure factor consists of several peaks: the quasielastic or $Q$-peak around $\omega=0$ and several inelastic peaks due to phonons and adsorbate vibrations of low frequency, for creation $(\hbar \omega<0)$ and annihilation $(\hbar \omega$ $>0)$ events. The above expression relating a cross section to a TCF implicitly assumes that it is possible to describe the collision of the projectile with a many-atom target as impulsive collisions with individual atoms, so that the cross section factors into quantities relating to a single projectile-atom collision times a quantity describing the dynamics of target atoms in the absence of the projectile. This is a well-known factorization $^{23}$ which can be validated for atom-atom interactions of short range, as expected here for He atom projectiles. The more general exact formal relation between TCFs and cross sections has been investigated and reviewed for atomic collisions in Ref. 18.

Although we are not presently incorporating diffusion effects in our model, it is possible to ascertain its role from the previous equations. In general, different length and time scales are involved in both motions: vibrations are localized inside adsorption potential minima and have short vibrational periods, while diffusion proceeds by jumping from one adsorption site to another between neighbors or even more distant sites at a slow rate. No correlation then exists between the rapid motion due to adsorbate vibrations and the slow diffusional motion. The $G$-function can be written as the convolution in position variables of the two contributions and, by the convolution theorem, the total intermediate scattering function would be the product of two Fourier transforms in wave vector variables, one coming from vibration and the other from diffusion. ${ }^{21}$ The total dynamic structure factor could then be expressed as the convolution in the frequency variable of the two corresponding dynamic structure factors. By considering the quasielastic (zero-phonon) and inelastic (one-phonon) contributions, the total dynamic structure factor can be expressed as a sum, each term coming from a different type of motion. If, on the contrary, both motions are coupled (for example, with increasing surface temperature, thermal energies can be higher than the diffusion barrier and this results in faster diffusion), then diffusion is assisted by vibrations which contribute to the quasielastic peak and a sum is no longer meaningful. ${ }^{24}$ Furthermore, if large friction coefficients are present this can lead to jump distances of the order of the substrate lattice spacing. In the limit of strong correlation, no detectable trace of vibrations is expected and only a broad quasielastic peak is observed.

On the other hand, anharmonic vibrational motions could also affect the shape and width of the spectral peaks when the thermal population of the corresponding high vibrational states is substantial. In our case, surface temperatures are low compared with characteristic vibrational temperatures of the adsorbate, and the population of the corresponding higher energy levels is expected to be small.

In any case, observed line shapes must be deconvoluted since the FWHM of the response function of the detector and the vibrational relaxation line shape are usually comparable.

\section{B. The excited damped harmonic oscillator model}

Our theoretical development starts from the driven damped harmonic oscillator model. First, a harmonic oscillator is a good starting point for a single, isolated adsorbate displaying a frustrated translational motion ( $T$-mode). Second, the reservoir is considered as a collection of independent oscillators at thermal equilibrium at the surface temperature, and thermal fluctuations are introduced in the reservoir, which feeds noise into the oscillator, leading to a dissipation or loss mechanism. And, third, the driving force can be assumed to derive from a repulsive $\mathrm{He}-A$ potential energy, with the motion of the projectile given by a classical trajectory function of time in an impulsive collision regime. For a He beam of low energy, the net effect of the scattering process can be visualized by means of the creation or annihilation of only one adsorbate vibrational quantum at a time of a given frequency and the so-called rotating wave approximation is acceptable. ${ }^{19}$ The oscillator with frequency $\omega_{0}$ is weakly and bilinearly coupled to the reservoir and loses energy to the reservoir; conversely, the fluctuations in the reservoir also couple back into the $T$-mode. With all of these ingredients, the total Hamiltonian can be written as follows

$$
H=H_{A}+H_{R}+H_{A R}+H_{\mathrm{He} A},
$$

where the Hamiltonian for the dynamical system is

$$
H_{A}=\hbar \omega_{0} a^{\dagger} a \text {. }
$$

The lattice reservoir Hamiltonian is expressed as the sum over normal modes $j$,

$$
H_{R}=\sum_{j} \hbar \omega_{j} b_{j}^{\dagger} b_{j}
$$

the interaction between the adsorbate and lattice is assumed to be bilinear as

$$
H_{A R}=\sum_{j} \hbar\left[\kappa_{j} a^{\dagger} b_{j}+\kappa_{j}^{\dagger} a b_{j}^{\dagger}\right]
$$

where the coupling coefficients $\kappa_{j}=c_{j} /\left(m_{j} \omega_{j}\right)^{1 / 2}$ are written in terms of the coupling strengths $c_{j}$ and the lattice normal mode effective masses $m_{j},{ }^{6}$ and finally the projectile driving interaction with the adatom is given by

$$
H_{\mathrm{He} A}=\hbar\left[f(t) a^{\dagger}+f^{*}(t) a\right] .
$$

In Eqs. (2.6)-(2.9), $a^{\dagger}$ and $a$ are the creation and annihilation operators describing the $T$-mode of the adsorbate and fulfilling the commutation relation $\left[a, a^{\dagger}\right]=1$, the normal mode operators $b_{j}$ and $b_{j}^{\dagger}$ satisfy the boson commutation relations $\left[b_{j}, b_{k}^{\dagger}\right]=\delta_{j k}$, the coefficients $\kappa_{j}$ account for the strength of their coupling, and $f(t)$ is the driving force. We are assuming an effective interaction in Eq. (2.8), partially 
mediated by electron-hole excitations, with the $\kappa_{j}$ coefficients large only for phonons which nearly conserve energy $\left(\omega_{j} \approx \omega_{0}\right)$ and momentum.

Cross sections can be obtained within the collisional TCF approach in the impulsive collision regime without reference to the driving Hamiltonian term, insofar the cross section can be factored, as mentioned, into an atom pair collisional factor and a time correlation factor for the target. However, some considerations can be made here about the role of the driving force. First, the attractive part of the potential does not play an important role in vibrational excitation problems, and it can be neglected. And second, the incident energy for the incoming particles is large compared to the quantum of vibrational excitation. A semiclassical description of the projectile-adsorbate interaction allows for an estimate of the collisional time and the duration of energy transfer. Thus, if $r$ is the distance between the He atoms and the center of mass of the adsorbate and their interaction is accepted to be exponentially repulsive, $H_{\mathrm{He} A}=C e^{-\alpha^{\prime} r}$, it can be easily shown ${ }^{25}$ that $f(t)=\alpha \operatorname{sech}^{2} \alpha t$ with $\alpha$ $=\alpha^{\prime} v_{i} \sin \theta_{i} / 2, v_{i}$ and $\theta_{i}$ being the incident velocity and angle of the probe particles, respectively. The parameter $\alpha$ gives the rate of energy exchange between the translational and frustrated translation motions. The hyperbolic function has the physically correct behavior at the asymptotic limits $t \rightarrow \pm \infty$ and is maximum at $t=0$ where the closest distance to the adsorbate is reached. This simple expression can be used to confirm that the collision is impulsive.

Several theoretical formulations could be followed within this model to obtain transition rates or cross sections. One of them is, for example, the density operator formulation ${ }^{19,26}$ which incorporates population and coherence relaxation rates and allows for a treatment of temperature effects. It is possible to follow alternative formulations of relaxation, and recent work ${ }^{13,14}$ analyze them in detail. Here we prefer to follow a quantum Langevin formulation ${ }^{19}$ to interpret the experimental results in a language similar to a classical Langevin treatment insofar the data adjust quite nicely to the classical damping simulations. Therefore, we are going to work with the equations of motion of operators in the Heisenberg picture of quantum mechanics. System fluctuations are smoothed out on a time scale during which the reservoir is correlated but not on a scale during which the system is damped. The standard procedure consists of solving the coupled equations for adsorbate and reservoir operators, by first solving for the reservoir equations and replacing the sums over reservoir modes with damping terms in the Heisenberg equations of motion for the adatom. A term could be added to describe the driving forces, but this is not needed in the TCF treatment.

Writing the general equation of motion of an operator $M$ as

$$
\frac{d M}{d t}=\frac{1}{i \hbar}[M, H]
$$

which can be used for both $M=a(t)$ and $M=b_{j}(t)$ operators, to derive their coupled equations with initial conditions $a(0)$ and $b_{j}(0)$, and with a given thermal distribution of the reservoir modes $j$. Solving formally for $b_{j}(t)$ and replacing in the equation for $a$, we have that

$$
\frac{d a}{d t}=-i \omega_{0} a-\sum_{j}\left|\kappa_{j}\right|^{2} \int_{0}^{t} d t^{\prime} a\left(t^{\prime}\right) e^{i \omega_{j}\left(t^{\prime}-t\right)}+F_{r}(t),
$$

where $F_{r}(t)$ is the Langevin operator for a noise source expressed as

$$
F_{r}(t)=-i \sum_{j} \kappa_{j} b_{j}(0) e^{-i \omega_{j} t}
$$

and Eq. (2.11) can be seen as a generalized quantum Langevin equation with a nonohmic friction given by

$$
\gamma\left(t^{\prime}-t\right)=\sum_{j}\left|\kappa_{j}\right|^{2} e^{i \omega_{j}\left(t^{\prime}-t\right)} .
$$

Under the so-called Wigner-Weisskopff approximation for this model, ${ }^{19}$ requiring a time evolution sufficiently long to ensure that the system has significantly decayed, it is a straightforward exercise to obtain the solution of the Heisenberg equation of motion for the annihilation operator of a damped oscillator with Hamiltonian given by Eqs. (2.5) $-(2.8)$ as

$$
\begin{aligned}
a(t)= & a(0) e^{-i\left(\omega_{0}+\Delta \omega\right) t-\gamma t / 2} \\
& -\sum_{j} \frac{\kappa_{j} e^{-i \omega_{j} t}\left[1-e^{i\left(\omega_{j}-\omega_{0}-\Delta \omega\right) t-\gamma t / 2}\right]}{\omega_{0}-\omega_{j}+\Delta \omega-i \gamma / 2} b_{j}(0) .
\end{aligned}
$$

Here the frequency shift $\Delta \omega$ and relaxation rate $\gamma$ have been defined so that

$$
\begin{aligned}
& -i \sum_{j} \frac{\left|\kappa_{j}\right|^{2}}{\left(\omega_{j}-\omega_{0}-i \epsilon\right)} \\
& \quad=-i \int d \omega_{j} g\left(\omega_{j}\right)\left|\kappa\left(\omega_{j}\right)\right|^{2} /\left(\omega_{j}-\omega_{0}-i \epsilon\right) \\
& \quad \simeq \gamma / 2+i \Delta \omega,
\end{aligned}
$$

where $g\left(\omega_{j}\right)$ is the density of normal modes of the reservoir at its temperature and $\epsilon \rightarrow 0$ is a small positive increment. After integration, the relaxation rate or friction coefficient is

$$
\gamma=2 \pi g\left(\omega_{0}\right)\left|\kappa\left(\omega_{0}\right)\right|^{2}
$$

and the shift $\Delta \omega$ is given by

$$
\Delta \omega=-P P \int_{-\infty}^{+\infty} \frac{g\left(\omega_{j}\right)\left|\kappa\left(\omega_{j}\right)\right|^{2}}{\omega_{j}-\omega_{0}} d \omega_{j},
$$

where $P P$ means the Cauchy principal value of the integral.

Notice that insofar the time integral in Eq. (2.11) simplifies for an instantaneous dissipation when $\gamma\left(t^{\prime}-t\right)=\gamma \delta\left(t^{\prime}\right.$ $-t$ ); this equation can then be rewritten as the Langevin-like equation

$$
\frac{d a}{d t}=-i \omega_{0} a-\frac{\gamma a}{2}+F_{r}(t)
$$


and the frequency shift dissappears. The term $\gamma a / 2$ now accounts for friction in the vibrational motion. The commutation rule for the creation and annihilation operators are now fulfilled only in the statistical sense since it is only the reservoir average of the commutator of $a$ and $a^{\dagger}$, over the thermal distribution of $j$ modes, which becomes independent of time and equal to one.

Properties of $F_{r}(t)$ are obtained quite straightforwardly when stated for thermal averages over the reservoir statistical distribution: zero mean $\left\langle F_{r}(t)\right\rangle_{R}=0$ and correlation function $\left\langle F_{r}^{\dagger}\left(t_{1}\right) F_{r}\left(t_{2}\right)\right\rangle_{R}=\gamma\left(t_{1}-t_{2}\right) \bar{n}$ (with a Fourier transform varying with frequency, corresponding to colored noise), where $\bar{n}$ is the Bose-Einstein distribution at temperature $T$ and vibrational energy quantum $\hbar \omega_{0} \cdot{ }^{19}$

As a consequence, we find from Eq. (2.14) that the reservoir averages of $a$ and $N=a^{\dagger} a$ are given by

$$
\begin{aligned}
& \langle a(t)\rangle_{R}=\langle a(0)\rangle_{R} \exp \left[-i\left(\omega_{0}+\Delta \omega\right) t-\gamma t / 2\right], \\
& \langle N(t)\rangle_{R}=\langle N(0)\rangle_{R} \exp (-\gamma t) .
\end{aligned}
$$

In what follows, we will assume that averages over the reservoir distribution have been already made.

\section{The $T$-mode line shape}

Starting from Eq. (2.4), the intermediate scattering function can also be expressed as

$$
I(\mathbf{K}, t)=\left\langle e^{-i \mathbf{K} \cdot \mathbf{R}(0)} e^{i \mathbf{K} \cdot \mathbf{R}(t)}\right\rangle,
$$

where the component $\mathbf{K}$ of the collisional momentum transfer along the surface is fixed by the experimental setup. The component $R_{K}(t)=u(t)$ of the parallel displacement of the adsorbate along $\mathbf{K}$ is the only coordinate that must be explicitly considered. For a damped harmonic oscillator, this onedimensional displacement can be written as

$$
\begin{aligned}
u(t)= & \left(\frac{\hbar}{2 M \omega_{T}}\right)^{1 / 2} \exp (-\gamma t / 2) \\
& \times\left[a \exp \left(-i \omega_{T} t\right)+a^{\dagger} \exp \left(i \omega_{T} t\right)\right],
\end{aligned}
$$

where $\omega_{T}=\omega_{0}+\Delta \omega$ and the algebra of vibrational operators can be used to calculate the scattering TCF. This can be done extending treatments in the literature (see, e.g., Ref. 6) to include dissipative effects. Starting with the identity

$$
\begin{aligned}
\exp [ & -i K u(0)] \exp [i K u(t)] \\
& =\exp \left\{\frac{1}{2} K^{2}[u(0), u(t)]\right\} \times \exp \{-i K[u(0)-u(t)]\}
\end{aligned}
$$

and using the well-known formula (see, e.g., Ref. 6)

$$
\langle\exp Q\rangle=\exp \left(\frac{1}{2}\left\langle Q^{2}\right\rangle\right)
$$

the scattering TCF with friction is given by

$$
\begin{aligned}
\langle\exp [ & -i K u(0)] \exp [i K u(t)]\rangle \\
= & \exp \left\{-K^{2}\left[\left\langle\left[(u(0)]^{2}\right\rangle\left(\frac{1+\exp (-\gamma t)}{2}\right)\right.\right.\right. \\
& -\langle u(0) u(t)\rangle]\}
\end{aligned}
$$

The displacement autocorrelation function appearing in Eq. (2.24) is easily obtained to be

$$
\begin{aligned}
\langle u(0) u(t)\rangle= & \frac{\hbar}{2 M \omega_{T}} \exp (-\gamma t / 2)\left[\left(1+\left\langle a^{\dagger} a\right\rangle\right)\right. \\
& \left.\times \exp \left(i \omega_{T} t\right)+\left\langle a^{\dagger} a\right\rangle \exp \left(-i \omega_{T} t\right)\right],
\end{aligned}
$$

where the Bose-Einstein population distribution is given at temperature $\beta=\left(k_{B} T\right)^{-1}$ by

$$
\left\langle a^{\dagger} a\right\rangle=\frac{1}{\exp \left(\hbar \omega_{T} \beta\right)-1}=\bar{n} .
$$

Therefore, the intermediate TCF given by Eq. (2.20) can be expressed, using the dimensionless quotient $\lambda^{2}$ $=\hbar K^{2} /\left(2 M \omega_{T}\right)$, as

$$
\begin{aligned}
I(K, t)= & \exp \left[-\lambda^{2}(2 \bar{n}+1)\left(\frac{1+\exp (-\gamma t)}{2}\right)\right] \\
& \times \exp \left\{\lambda ^ { 2 } \operatorname { e x p } ( - \gamma t / 2 ) \left[(\bar{n}+1) \exp \left(i \omega_{T} t\right)\right.\right. \\
& \left.\left.+\bar{n} \exp \left(-i \omega_{T} t\right)\right]\right\} .
\end{aligned}
$$

This expression can be rewritten as

$$
\begin{aligned}
I(K, t)= & \exp \left[-\lambda^{2} \operatorname{coth}\left(\frac{\hbar \omega_{T} \beta}{2}\right)\left(\frac{1+\exp (-\gamma t)}{2}\right)\right] \\
& \times \exp \left\{\lambda^{2} \operatorname{cosech}\left(\frac{\hbar \omega_{T} \beta}{2}\right) \exp (-\gamma t / 2)\right. \\
& \times\left[\exp \left(i \omega_{T} t+\frac{\hbar \omega_{T} \beta}{2}\right)\right. \\
& \left.\left.+\exp \left(-i \omega_{T} t-\frac{\hbar \omega_{T} \beta}{2}\right)\right]\right\}
\end{aligned}
$$

and further using the expansion

$$
\exp \left[\frac{1}{2} y\left(x+x^{-1}\right)\right]=\sum_{n=-\infty}^{\infty} x^{n} I_{n}(y),
$$

where $I_{n}$ is the modified Bessel function of the first kind, we arrive at the compact expression

$$
\begin{aligned}
I(K, t)= & \exp \left\{-\lambda^{2} \operatorname{coth}\left(\frac{\hbar \omega_{T} \beta}{2}\right)\left(\frac{1+\exp (-\gamma t)}{2}\right)\right\} \\
& \times \sum_{n=-\infty}^{\infty} \exp \left(i \omega_{T} t n+\frac{\hbar \omega_{T} \beta n}{2}\right) I_{n}[y(t)],
\end{aligned}
$$

where

$$
y(t)=\lambda^{2} \operatorname{cosech}\left(\frac{\hbar \omega_{T} \beta}{2}\right) \exp (-\gamma t / 2) .
$$


The remaining step linking these equations to cross sections involves the numerical Fourier transform of $I(K, t)$ to obtain the dynamic structure factor $S(K, \omega)$ given by Eq. (2.4), which now includes dissipative effects through the friction coefficient.

In order to gain more insight on the physical problem tackled here, it is interesting to write an analytical expansion for the dynamic structure factor and to discuss the meaning of the different contributions to its close formula. From Eq. (2.27), it is clear that the factor independent of time can be considered as a Debye-Waller or global attenuation factor written as

$$
e^{-2 W} \equiv \exp \left[-\lambda^{2}(\bar{n}+1 / 2)\right] .
$$

After this we have three exponential factors with arguments involving $\gamma$ and $\omega_{T}$. If a Taylor series expansion is used for each exponential factor and the Fourier transform in time is applied to Eq. (2.27), we arrive after simple manipulations to

$$
\begin{aligned}
(K, \omega)= & e^{-2 W} \sum_{n, m, l=0}^{\infty} \frac{(-1)^{l} \lambda^{2(n+m+l)} \bar{n}^{n}(\bar{n}+1)^{m}(\bar{n}+1 / 2)^{l}}{S n ! m ! l !} \\
& \times \frac{(n+m+2 l) \gamma}{\left[\omega-(n-m) \omega_{T}\right]^{2}+[(n+m+2 l) \gamma / 2]^{2}} .
\end{aligned}
$$

Several comments are in order. The line shape is a combination of an infinite number of Lorentzian functions, which is obviously unlike a single Lorentzian function. The quasielastic peak is obtained from inelastic events with a zero net energy balance $(n=m)$ and the inelastic adsorbate vibrational peaks result from inelastic events with $n \neq m$. The global shift and broadening of the total line shape is the result of many partial shifts and broadenings. The temperature dependence of the line shape comes from the Debye-Waller factor and, as we will show later, also from $\omega_{T}$ and $\gamma$.

\section{THE VIBRATIONAL RELAXATION OF THE $\mathrm{Na}, \mathrm{CO} / \mathrm{Cu}(001)$ AND CO/Pt(111) SYSTEMS}

Inelastic $\mathrm{He}$ atom scattering has been used to study the low-frequency vibrational motions of $\mathrm{Na}$ on $\mathrm{Cu}(001)$, $\mathrm{CO}$ on $\mathrm{Cu}(001)$, and $\mathrm{CO}$ on $\mathrm{Pt}(111)$ with low coverage, using a beam with projectile energy between 10 and $20 \mathrm{meV} \cdot{ }^{10} \mathrm{In}$ particular, for the $\mathrm{Na} / \mathrm{Cu}(001)$ system, experimental results for the collisional excitation of the $T$-mode show a line shape with a peak shift and a width dependent on the surface temperature going from $50 \mathrm{~K}$ to $200 \mathrm{~K}$. Experimental values were then extrapolated to $T=0 \mathrm{~K}$, and gave the excitation energy $\hbar \omega_{0}=6 \mathrm{meV}$ for the adatom $T$-vibration and a friction rate constant $\gamma=0.1 \omega_{0}$. To relate this system to our model, it is instructive to estimate the orders of magnitude of the time $\mathrm{He}$ atoms take to vibrationally excite $\mathrm{Na}$, namely, the collision time and the average rate of diffusion, and to compare them with typical response times for the lattice phonons and the electron-hole lifetimes. Thus we have that the duration $\Delta t_{\text {exc }}$ of the energy transfer process leading to the $0 \rightarrow 1$ transition of the vibrating $\mathrm{Na}$ can be estimated from $\Delta E \Delta t=\hbar$, with $\Delta E=6 \mathrm{meV}$ as measured; this gives a value $\Delta t_{\text {exc }} \simeq 110 \mathrm{fs}$. A lower bound to the collision time $\Delta t_{\text {col }}$ is estimated from the range $R_{\mathrm{HeNa}} \simeq 4 \AA$ of the projectile-adatom interaction and the velocity $v_{\mathrm{He}}$ of the $\mathrm{He}$ beam by means of $\Delta t_{\text {col }}>R_{\mathrm{HeNa}} / v_{\mathrm{He}} \simeq 425 \mathrm{fs}$, and of order 1 ps. The third relevant quantity, the average rate of diffusion $k_{d i f}=1 / \tau_{M F P T}$ follows from our calculations ${ }^{27}$ of the mean first passage time which gave the value $\tau_{M F P T} \simeq 3427 \mathrm{fs}$ at $T=200 \mathrm{~K}$. First, it is clear that diffusion is slow compared with the time of collisional energy transfer and can be presently ignored. Second, one can compare excitation and collision times with typical $e-h$ relaxation times, which are of the order of $20 \mathrm{fs}$ in $\mathrm{Cu}$ (Refs. 28-31) for direct $e-h$ relaxation (the relevant decay mechanism in our case), to conclude that $e-h$ excitations play a role in the relaxation phenomenon only during a short interval within the overall interaction time. A similar physical justification can be applied to the remaining two systems of present interest, $\mathrm{CO} /$ $\mathrm{Cu}(001)$ and $\mathrm{CO} / \mathrm{Pt}(111)$. Our theoretical model, derived under the assumption that relaxation is dominated over long times by lattice phonon, ${ }^{16}$ can be applied to all of three systems. Moreover, it has been shown ${ }^{4,32}$ that the $e$ - $h$ pair contribution is independent of temperature if adsorbate vibrations are harmonic and the corresponding coupling is linear, while the measured temperature dependences point to a role for lattice vibrations.

By assuming a simple Debye model for lattice vibrations of $\mathrm{Cu}$ and $\mathrm{Pt}$, the density of vibrational modes per unit frequency is given by

$$
g_{0}(\omega)=g_{D}(\omega)=18 \pi N \omega^{2} / \omega_{D}^{3}
$$

with $g_{0}(\omega)=0$ for $\omega>\omega_{D}$, where $N$ is the number of lattice atoms and $\omega_{D}$ is the Debye cutoff frequency. We have used Debye energies $\epsilon_{D}=\hbar \omega_{D}$ equal to $27.57 \mathrm{meV}$ for $\mathrm{Cu}(001)$ and $19.82 \mathrm{meV}$ for $\mathrm{Pt}(111)$. The corresponding Debye temperatures $T_{D}$ are $320 \mathrm{~K}$ and $230 \mathrm{~K}$. The experimental surface temperature range is between $50 \mathrm{~K}$ and $200 \mathrm{~K}$, below $T_{D}$ and therefore the probability of multiphonon events is very low. When the lattice is at temperature $T$, the population of lattice vibrational modes should reflect the appearance of the thermal lattice excitation. In this case the transfer of energy from the excited adsorbate to the lattice involves additional initial lattice states and a probability of excitation proportional to the initial population $\bar{n}(\omega, T)$ for each mode of lattice frequency $\omega$. Therefore the density $g$ must be multiplied by a factor $1+\bar{n}$ (a thermal population factor), which changes it to

$$
g_{T}(\omega)=g_{0}(\omega)(1+\bar{n})=g_{0}(\omega) \frac{\exp \left(\hbar \omega / k_{B} T\right)}{\exp \left(\hbar \omega / k_{B} T\right)-1} .
$$

The other quantity appearing in calculations is the coupling $\kappa(\omega)$ between the adsorbate vibration and the lattice modes of frequency $\omega$. As indicated above, this is to be considered as an effective coupling which conserves energy and momentum and displays significant values around the proper frequency $\omega_{0}$ of the adsorbate. Here we need it only in the neighborhood of $\omega_{0}$, as seen in Eq. (2.15). Including now the temperature dependence, we find that

$$
\gamma_{T}(\omega)=2 \pi g_{T}(\omega)|\kappa(\omega)|^{2}
$$

and 

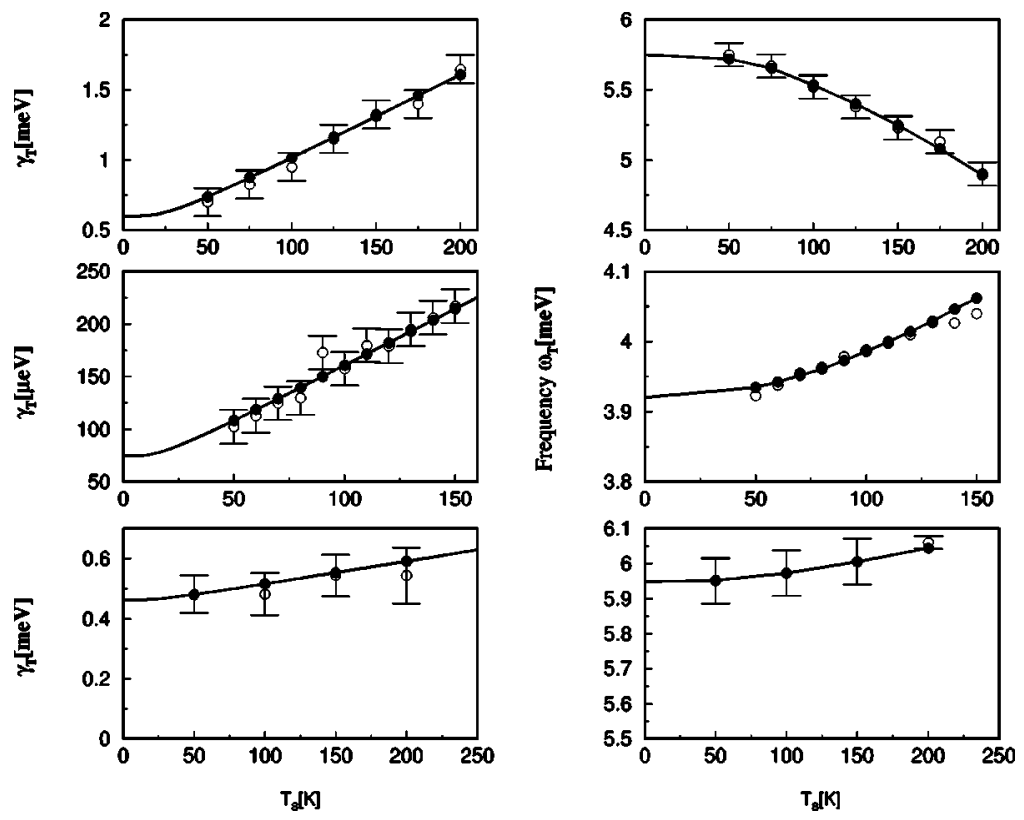

FIG. 1. Broadenings (FWHM) $\gamma_{T}$ and vibrational frequencies $\omega_{T}$ of the inelastic $T$-peak (black circles with solid lines) vs surface temperature $T_{s}$ and their comparison with experimental points (white circles) for the $\mathrm{Na} / \mathrm{Cu}(001)$ (top), $\mathrm{CO} / \mathrm{Cu}(001)$ (medium) and $\mathrm{CO} /$ $\mathrm{Pt}(111)$ (bottom) systems. The experimental conditions for each system are given in the literature (Ref. 10).

$$
\Delta \omega(T)=-P P \int_{0}^{\omega_{D}} d \omega \frac{g_{T}(\omega)|\kappa(\omega)|^{2}}{\omega-\omega_{0}} .
$$

The latter is the temperature dependent frequency shift, while $\omega_{0}+\Delta \omega(T=0)$ is seen as the value remaining at $0 \mathrm{~K}$ and could be referred to as the residual shift, insofar it is present even at $0 \mathrm{~K}$. The friction coefficient is not zero in this limit, because the excited adsorbate can yet transfer its energy to the lattice, to induce excitations from the zero-point motions of the lattice modes, and can also create e-h excitations in the electron distribution.

For the purpose of evaluating the integral, we have parametrized the positive quantity $|\kappa(\omega)|^{2}$, locally around $\omega$ $=\omega_{0}$ with the following linear form (Taylor series expansion up to first order):

$$
|\kappa(\omega)|^{2}=\left[p+q\left(\omega-\omega_{0}\right)\right] / N .
$$

The $p$ and $q$ parameters provide information about the strength of the adsorbate-lattice vibrational couplings and about the masses of the lattice modes, for lattice frequencies close to the adsorbate vibrational frequency.

Values of $p$ and $q$ can be obtained within a fitting procedure to the experimental frequency shift and width of the $T$-peak. First, starting with Eq. (3.36) and at $\omega=\omega_{0}$, we calculate $p$ from the experimental broadening at a given temperature, here chosen to be $150 \mathrm{~K}$. It has been observed that the $p$ parameter depends very weakly on the surface temperature for the three systems studied. For the same temperature $(150 \mathrm{~K})$ and from Eq. (3.37) and the resulting $p$ value, we calculate the $q$ parameter. These two values for $p$ and $q$ are the ones used for the whole range of surface temperatures. This procedure is followed for the three systems studied here. We have used the following values for the adsorbate frequencies: $\quad \hbar \omega_{0}(\mathrm{Na} / \mathrm{Cu})=6 \mathrm{meV}, \quad \hbar \omega_{0}(\mathrm{CO} / \mathrm{Cu})$ $=3.94 \mathrm{meV}$, and $\hbar \omega_{0}(\mathrm{CO} / \mathrm{Pt})=5.94 \mathrm{meV}$. The sign of the $q$ parameter is a mere consequence of this procedure; it is not forced a priori. As $q$ is proportional to the first derivative at $\omega_{0}$, its sign relates to the slope of $\kappa(\omega)$ around $\omega_{0}$ and pro- vides some information on the shape of the vibrational coupling around the frequency probed by the adsorbate vibrations. This procedure results in the following $(p, q)$ values for each system: $\left(2.31 \times 10^{-7}\right.$ a.u. $^{-2},-5.80 \times 10^{-5}$ a.u. $\left.^{-1}\right)$ for $\mathrm{Na} / \mathrm{Cu}(001) ;\left(6.44 \times 10^{-8}\right.$ a.u. $^{-2}, 1.58 \times 10^{-5}$ a.u. $\left.^{-1}\right)$ for $\mathrm{CO} / \mathrm{Cu}(001)$, and $\left(1.10 \times 10^{-8}\right.$ a.u. $^{-2}, 3.98 \times 10^{-6}$ a.u. $\left.^{-1}\right)$ for $\mathrm{CO} / \mathrm{Pt}(111)$. We plot in Fig. 1 the corresponding FWHM broadenings, found to be numerically close to $\gamma_{T}$, and vibrational frequencies $\omega_{T}$ versus the surface temperature $T_{s}$ for the three systems, from top to bottom. Open circles with error bars correspond to the experimental values and the solid lines linking black circles to our fittings. The agreement in the three cases is quite good in the surface temperature range covered by the experiments. The observed redshifts or blueshifts are well reproduced with the change of sign in the $q$ value as one goes from $\mathrm{Na}$ to $\mathrm{CO}$. An additional interesting feature of this theory is that it provides an extrapolating law at zero surface temperature. The corresponding extrapolated broadenings and shifts differ only little from the ones proposed by the experimental work. ${ }^{10}$ At higher temperatures, anharmonicity effects should play an increasing role and our model should be extended to include such effects.

For the $\mathrm{Na} / \mathrm{Cu}(001)$ system, and using the $p$ and $q$ values obtained before, we have calculated the intermediate scattering function $I(K, t)$ and the line shapes from the dynamical scattering function $S(K, \omega)$. A single Lorentzian function has been assumed to play a dominant role. Figure 2 shows the intermediate scattering function $I$ versus both time $t$ and physical values of the friction parameter $\gamma$, for a wave number transfer of $K=1.43 \AA^{-1}$ and $T=150 \mathrm{~K}$, with the direction of $\mathbf{K}$ along the [100] azimuth, a diagonal of the surface lattice. Only three terms $n=-1,0,1$ in Eq. (2.30) have been included, as needed for convergence. The oscillation frequency of such a function is given by $\omega_{T}$. As the friction increases, the decay of the scattering function becomes more pronounced and the signature of the $T$-mode starts disappearing. Figure 3 shows the corresponding nu- 


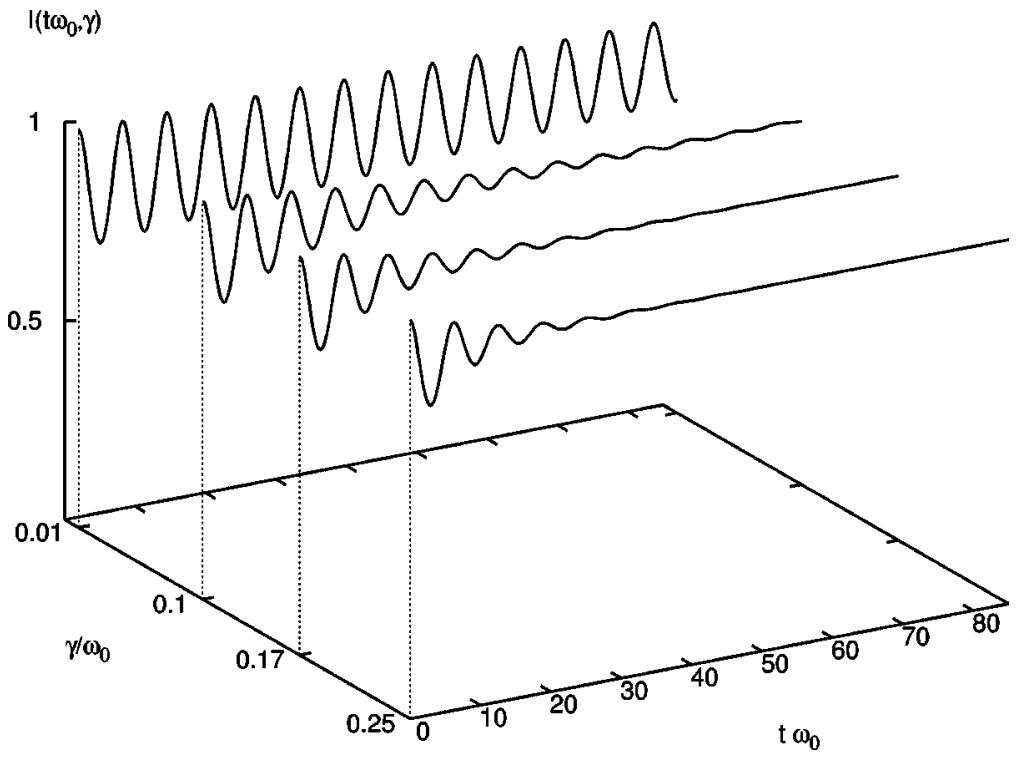

FIG. 2. The intermediate scattering function $I$ vs both time $t$ and physical values of the friction coefficient $\gamma$, in reduced units using the adatom frequency $\omega_{0}$, for a wave number transfer of $K=1.43 \AA^{-1}$ and $T=150 \mathrm{~K}$, with the direction of $\mathbf{K}$ along the [100] azimuth, a diagonal of the surface lattice. merical values of the dynamic structure function versus frequency and friction, and displays the two inelastic peaks typical of time-of-flight transfer, for excitation and deexcitation of a single vibrational quantum of the adsorbate. For clarity, the large quasielastic peak, coming from $n=m$, has been removed from the figure. As expected, the line shapes are seen to decrease in height and broaden as the friction increases. It follows from Eq. (2.33) that the partial line shapes contributing to the total line shape are shifted $(n$ $-m) \omega_{T}$ and broadened $(n+m+2 l) \gamma_{T} / 2$. If only the first harmonic of the $T$-mode is excited, then $n= \pm 1, m=0$, and $l=0$, and insofar contributions due to $n-m=1$ for all other $n$ and $m$ are not considered, the shift is given by $\omega_{T}$ from Eq. (3.37). The corresponding broadening, to the lowest order, is given by $\gamma_{T}$ in Eq. (3.36).

\section{CONCLUSION}

In this work, we have derived an extension of the collisional TCF treatment to include dissipative effects within the damped harmonic oscillator model, starting from a description of the medium (metal substrate) as a collection of oscillators with boson-like excitations. We have also provided a plausible interpretation for the temperature dependence of line shapes of the frustrated translational, or $T$-peak, based on the energy transfer from an excited adsorbate to the lattice, meaningful even at $T=0 \mathrm{~K}$. The agreement found with the experimental results in the measured surface temperature range is very good when the friction parameter is allowed to depend on the frequency of the adsorbate and the surface temperature, and when a Debye model for the substrate vibrations is assumed. No explicit effective adsorbate-substrate interaction potential model has been required in the model, only the strength of the frequency dependent coupling between vibrational modes.

The magnitudes of the $p$ and $q$ parameters introduced by our model are related to the strength $c_{j}$ of vibrational couplings and to the effective masses and frequencies of vibrational modes $j$. Further interpretation would require a de-

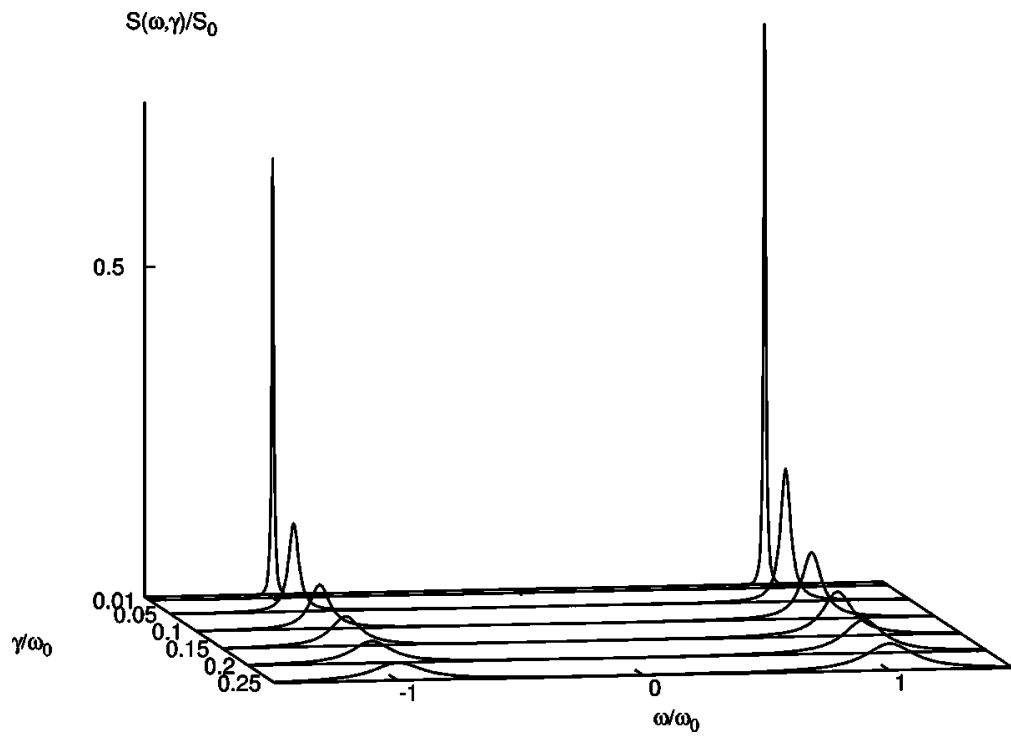

FIG. 3. Relative numerical values of the dynamic structure function $S$ vs frequency and friction corresponding to the intermediate scattering functions from Fig. 2 are displayed. The two inelastic $T$-peaks typical of time-offlight measurements for transfer, for excitation and deexcitation of a single quantum are observed. For clarity, the large quasielastic peak, coming from $n=m$, has been removed from the figure. 
tailed treatment of the lattice dynamics including the adsorbate motion, which is outside the scope of this work. In fact, the present treatment shows how our analysis could extract information from experimental work, to be compared with theory. For example, the sign of $q$ relates to the slope of $\kappa(\omega)$ around $\omega_{0}$ and provides some information on the shape of the vibrational coupling around the frequency probed by the adsorbate vibrations. Furthermore, an extrapolating law at $T=0 \mathrm{~K}$ is provided for the calculation of intrinsic (or as we wrote, residual) shifts and broadenings. Our theoretical model suggests that the limiting values arise primarily from e-h excitations, as the adsorbate vibrations relax at $T \rightarrow 0$. Our qualitative consideration of collisional and relaxation times suggest that the phonon and $e-h$ relaxation mechanisms are not parallel but sequential, with $e-h$ followed by phonon excitations, so that the total relaxation rate $\gamma_{\text {tot }}$ is not simply a sum of the rates $\gamma_{p h}$ and $\gamma_{e-h}$, but is instead a rate dominated at long times by $\gamma_{p h} \cdot{ }^{16}$

Alternative explanations using anharmonic vibration (see, for example, Ref. 15) and/or $e$ - $h$ metal excitations $s^{4,10}$ have been proposed and discussed in the literature to interpret experimental measurements with varying temperature. However, it seems that lattice phonons give a consistent explanation of the considered dynamic structure factor measurements. The approach proposed in this work implicitly assumes colored instead of white noise to describe dissipative behavior, insofar our $\gamma\left(t-t^{\prime}\right)$ is not equal to an instantaneous rate $\gamma \delta\left(t-t^{\prime}\right)$, and the Fourier transform of the time correlation function of the noise source is not a constant. ${ }^{33}$ More accurately, a general treatment of both quasielastic and inelastic atom scattering processes over the whole range of temperatures would consider phonon and $e-h$ excitations as well as anharmonic effects and would include colored noise, to ascertain which of all these factors is predominant within each surface temperature range and for different substrate metals.

\section{ACKNOWLEDGMENTS}

This work was supported in part by DGCYT (Spain) under Contract No. BFM2001-2179. J.L.V. and R.G. would like to thank the Ministry of Science and Technology (Spain) for a predoctoral grant and a Ramón y Cajal Contract, respectively. The work of D.A.M. was supported by the National Science Foundation of the USA.
${ }^{1}$ For a review see, for example, F. Hofmann and J. P. Toennies, Chem. Rev. (Washington, D.C.) 96, 1307 (1996).

${ }^{2}$ N. N. J. Persson and E. Zaremba, Phys. Rev. B 31, 1863 (1985).

${ }^{3}$ J. W. Gadzuk and A. C. Luntz, Surf. Sci. 144, 429 (1984).

${ }^{4}$ J. C. Tully, Annu. Rev. Phys. Chem. 51, 153 (2000).

${ }^{5}$ M. Bée, Quasielastic Neutron Scattering (Adam Hilger, Bristol, 1988).

${ }^{6}$ W. Marshall and S. W. Lovesey, Theory of Thermal Neutron Scattering (Oxford University Press, New York, 1971).

${ }^{7}$ H. Ibach and D. L. Mills, Electron Energy Loss Spectrsocopy and Surface Vibrations (Academic, New York, 1982).

${ }^{8}$ Y. J. Chabal, Surf. Sci. Rep. 8, 211 (1988).

${ }^{9}$ A. Lahee, J. P. Toennies, and C. Wöll, Surf. Sci. 177, 371 (1986).

${ }^{10}$ A. Graham, F. Hofmann, and J. P. Toennies, J. Chem. Phys. 104, 5311 (1996); A. P. Graham, F. Hofman, J. P. Toennies, L. Y. Chen, and S. C. Ying, Phys. Rev. B B56, 10567 (1997); A. Graham and J. P. Toennies, Europhys. Lett. 42, 449 (1998).

${ }^{11}$ A. M. Levine, M. Shapiro, and E. Pollak, J. Chem. Phys. 88, 1959 (1988).

${ }^{12}$ J. S. Bader, B. J. Berne, E. Pollak, and P. Hänggi, J. Chem. Phys. 104, 1111 (1996)

${ }^{13}$ V. May and O. Kuhn, Charge and Energy Transfer Dynamics in Molecular Systems (Wiley-VCH, Berlin, 2000), Chap. 4.

${ }^{14}$ D. Kohen and D. J. Tannor, Adv. Chem. Phys. 111, 219 (2000).

${ }^{15}$ R. Guantes, J. L. Vega, S. Miret-Artés, and E. Pollak, J. Chem. Phys. 120, 10768 (2004).

${ }^{16}$ D. A. Micha, J. L. Vega, R. Guantes, and S. Miret-Artes, Phys. Rev. Lett. (submitted).

${ }^{17}$ E. Vilallonga and D. A. Micha, J. Chem. Phys. 79, 3794 (1983).

${ }^{18}$ D. A. Micha and E. Vilallonga, Adv. Chem. Phys. 84, 1 (1993).

${ }^{19}$ W. H. Louisell, in Quantum Statistical Properties of Radiation (Wiley, New York, 1973).

${ }^{20}$ A. C. Levi, R. Spadacini, and G. E. Tommei, Surf. Sci. 121, 504 (1982).

${ }^{21}$ J. W. M. Frenken, and B. J. Hinch, in Helium Atom Scattering from Surfaces, Springer Series in Surface Sciences Vol. 27, edited by E. Hulpke (Springer-Verlag, Berlin, 1992), p. 287.

${ }^{22}$ D. McQuarrie, Statistical Mechanics (Harper \& Row, New York, 1976).

${ }^{23}$ L. Van Hove, Phys. Rev. 95(1), 249 (1954).

${ }^{24}$ J. L. Vega, R. Guantes, and S. Miret-Artés, J. Phys.: Condens. Matter 16, S2879 (2004).

${ }^{25}$ D. Rapp, J. Chem. Phys. 32, 735 (1960).

${ }^{26}$ K. Blum, Density Matrix Theory and Applications (Plenum, New York, 1981).

${ }^{27}$ J. L. Vega, R. Guantes, and S. Miret-Artés, Phys. Chem. Chem. Phys. 4, 4985 (2002); R. Guantes, J. L. Vega, S. Miret-Artés, and E. Pollak, J. Chem. Phys. 119, 2780 (2003).

${ }^{28}$ J. A. Prybyla, H. W. K. Tom, and G. D. Aumiller, Phys. Rev. Lett. 68, 3737 (1998).

${ }^{29}$ H. Guo, P. Saalfrank, and T. Seideman, Prog. Surf. Sci. 62, 239 (1999).

${ }^{30}$ D. A. Micha, A. Santana, and A. Salam, J. Chem. Phys. 116, 5173 (2002).

${ }^{31}$ E. Knoesel, A. Hotzel, and M. Wolf, Phys. Rev. B 57, 12812 (1998).

${ }^{32}$ B. N. J. Persson and J. W. Gadzuk, Surf. Sci. 410, L779 (1998).

${ }^{33}$ R. Kubo, M. Toda, and N. Hashitsume, Statistical Physics II, 2nd ed. (Springer, Berlin, 1991). 